\title{
Synthesis of electro-optically active polymer composite of poly[2,2'-bis(3,4-ethylenedioxythiophene)-alt-fluorene]/ hydroxypropyl cellulose showing liquid crystal structure
}

\author{
N. Eguchi, K. Kawabata, H. Goto* \\ Faculty of Pure and Applied Sciences, Division of Materials Science, University of Tsukuba, Tsukuba, 305-8573 Ibaraki \\ Japan
}

Received 28 December 2016; accepted in revised form 15 May 2017

\begin{abstract}
Electrochemical preparation of a composite consisting of poly[2,2'-bis(3,4-ethylenedioxythiophene)-alt-fluorene $]$ and hydroxypropyl cellulose (PEFE/HPC) was carried out. We conducted electrochemical polymerization of poly[2,2'bis(3,4-ethylenedioxythiophene)-alt-fluorene] (EFE) as a monomer in a lyotropic liquid crystal of HPC. We used an organic solvent instead of water for lyotropic liquid crystal medium to expand the possibility of the range of monomers, although water is usually employed as a solvent for HPC for showing liquid crystallinity. Here, we employed $N, N$-dimethylformamide (DMF) as a solvent for HPC. Electrochemical polymerization in the polymer liquid crystal was carried out to obtain a polymer film with liquid crystal order. The polymer film thus prepared exhibited optical activity. Fourier transfer infrared (FT-IR) absorption spectroscopy reveals that the film is a composite consisting of HPC and polymer. The composite PEFE/HPC thus prepared in HPC/DMF system showed electrochromism.
\end{abstract}

Keywords: polymer composites, electrochemical polymerization, electrochromism, conductive polymer, hydroxypropyl cellulose

\section{Introduction}

Electrochemical polymerization is a widely used technique to produce electrochromic materials [1-4]. Electric field is applied between an anode and a cathode in an electrolyte containing a monomer for performing electrochemical polymerization. Electrochemical polymerization proceeds at the surface of the anode, and a resultant conjugated polymer is deposited on the electrode. Todate, we have carried out electrochemical polymerization of conjugated polymer in thermotropic liquid crystal [5]. The polymer prepared in low molecular weight liquid crystal shows liquid crystal like structures because of the transcription of the liquid crystal order from the liquid crystal matrix during the polymerization process. The polymer itself contains no liquid crystal molecules because of phase separation during the polymerization reaction. In this polymerization process, liquid crystal acts as a template. Although the monomers have no optical activity, the resulting polymers prepared in chiral liquid crystals shows atropisomerism due to the occurrence of chirality transcription from molecular to macroscopic level. We referred to this method as 'asymmetric electrochemical polymerization' [6]. In this research, 2,7-bis(3,4-ethylenedioxythiophen-2-yl)fluorene (EFE), synthesized by our laboratory, was employed as a monomer, and the electrochemical preparation of poly[2,2'-bis(3,4-ethylenedioxythiophene)-alt-fluorene] (PEFE) was carried out. PEFE is known as an electrochromic material [7]. We have carried out electrochemical polymerization of EFE in a thermotropic liquid crystal with low molecular weight (hexyl cyanobiphenyl, $6 \mathrm{CB}$, thermotropic liquid crystal) as our previous

*Corresponding author, e-mail: gotoh@ims.tsukuba.ac.jp (C) BME-PT 
research and confirmed the helical order of the product derived from matrix cholesteric liquid crystal [8]. The polymer thus obtained shows laser diffraction derived from transcription of periodic structure of cholesteric liquid crystal. However, $6 \mathrm{CB}$ is expensive and has a certain temperature range in which it shows mesophase. Recently, we carried out electrochemical polymerization in hydroxypropyl cellulose (HPC) as a liquid crystal electrolyte in place of 6CB. HPC is a low cost semi-natural polymer with chirality. In previous research, some conjugated polymers were electrochemically synthesized in HPC liquid crystal $[9,10]$.

In the present study, we conduct electrochemical polymerization of EFE (monomer) in HPC. The product thus obtained is a polymer composite consisting of PEFE and HPC with no fibrillar structure, although PEFE prepared in 6CB shows fibrillar structure [8]. This electrochemical polymerization yields an electroactive polymer composite. We report the possibility of electrochemical polymerization in natural polymer as electrolyte solution to produce electrooptically active chiral polymer composite.

\section{Experimental}

\subsection{Materials}

EFE was previously synthesized [12]. Hydroxypropyl cellulose (HPC) was obtained from Wako Pure Chemical Industries, Ltd. (TCI, Japan) and used without further purification. Viscosity of the $\mathrm{HPC}$ is $2.0-.9 \mathrm{mPa} \cdot \mathrm{s}\left(2 \mathrm{wt} \%, 20^{\circ} \mathrm{C}\right)$. Tetrabutylammonium perchlorate (TBAP) was obtained from TCI (Japan) and used without further purification. $N, N$ dimethylformamide (DMF) was obtained from Nacalai Tesuque (Japan) and used without further purification.

\subsection{Electrochemical polymerization}

Electrochemical polymerization of EFE [8, 11], was carried out in hydroxypropyl cellulose (Figure 1). The chemical structure of hydroxypropyl cellulose is shown in Figure 2. Constituents of electrolyte solution are listed in Table 1. Various concentration solutions of HPC/ DMF were prepared and visually checked for liquid crystallinity. HPC employed in this study shows mesophase at $\sim 60 \mathrm{wt} \%$ in DMF. Phase diagram of HPC/ DMF has been reported previously [12].

First, EFE and TBAP (supporting salt) were dissolved in DMF. Next, hydroxypropyl cellulose was added to the solution containing supporting salt and
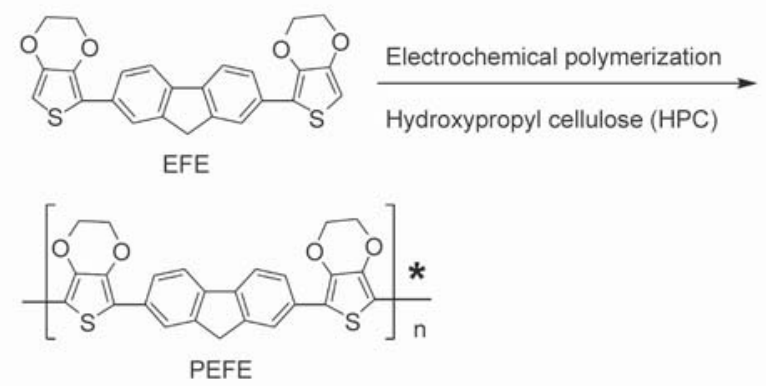

Figure 1. Electrochemical polymerization in hydroxypropyl cellulose/ $N, N$-dimethyl formamide (HPC/DMF). $*$ - chiral.

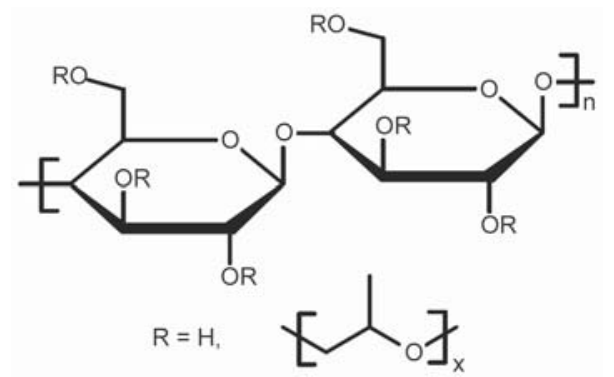

Figure 2. Chemical structure of hydroxypropyl cellulose (HPC)

the monomer. The solution was vigorously stirred mechanically for $1 \mathrm{~min}$ at room temperature. The solution was placed for $24 \mathrm{~h}$ to dissolve HPC homogeneously in DMF. Liquid crystallinity of the concentrated HPC solution was confirmed with visual inspection through iridescent color of the solution. EFE was pre-oriented in the helical environment of lyotropic HPC. Then, electrochemical polymerization was carried out by using the sandwiched cell method (two ITO glass electrodes, ITO = indium tin oxide) developed by our group previously. Direct current voltage of $2.5 \mathrm{~V}$ was applied across the cell for $30 \mathrm{~min}$ at room temperature. A polymer film was deposited onto the anode side. After completion of the electrochemical polymerization, the sandwiched cell was soaked in distilled water to remove the residual HPC from the film surface. After ca. $24 \mathrm{~h}$, the sandwich cell was disassembled and the polymer film was washed with distilled water, and acetone to obtain a smooth film deposited on the ITO coated glass. PEFE thus obtained is abbreviated as PEFE/HPC. To compare with standard PEFE film (pure PEFE), electrochemical polymerization of EFE in no HPC liquid crystal electrolyte solution was carried out using cyclic voltammetry scan method. An ITO coated glass, a platinum wire, and an $\mathrm{Ag} / \mathrm{Ag}^{+}$electrode were used as the working, counter, and reference electrodes, respectively. The electrolyte solution contained EFE 
Table 1. Constituents of electrolyte solution and monomer

\begin{tabular}{|l|l|l|l|}
\hline Monomer & Supporting salt & Liquid crystal matrix & Solvent \\
\hline (DMF, $403.4 \mathrm{mg})$
\end{tabular}

12,7-bis(3,4-ethylenedioxythiophen-2-yl)fluorene (EFE) [10].

${ }^{2}$ Tetrabutylammonium perchlorate.

${ }^{3}$ Hydroxypropyl cellulose.

and TBAP (Table 1). Electrochemical preparation of PEFE produced a film deposited on the ITO coated glass. To remove DMF (solvent), TBAP (supporting salt), and unreacted monomer, the product on the ITO was washed with acetone and distilled water thoroughly.

\subsection{Measurements}

Optical texture observations were conducted with an ECRILS LV 100 high-resolution polarizing microscope (Nikon). Fourier transform infrared absorption spectroscopy measurement ( $\mathrm{KBr}$ method) was carried out with an FT-IR 4600 (Jasco) at room temperature. UV-vis absorption spectra was obtained with V-630 (Jasco). Cyclic voltammetry (CV) measurements were carried out with a $\mu$ AUTOLAB TYPE III (ECO Chemie, the Netherlands). Circular dichroism (CD) spectroscopy measurements were carried out with a J-720 (Jasco).

\section{Results and discussion}

\subsection{POM observation}

The polymer film prepared in DMF with no HPC liquid crystal electrolyte solution shows no texture, Figure 3. The polymer film thus prepared in HPC liquid crystal shows a characteristic texture under polarizing optical microscopy (POM), Figure 4. The image was derived from cholesteric liquid crystal of helical aggregation, indicating transcription of helical aggregation structure of HPC occurred during the polymerization process. The electrochemical polymerization in the HPC liquid crystal solution gave a helical structure to PEFE. In other words, HPC functioned as a helical template in the electrochemical polymerization.

\subsection{FT-IR}

The FT-IR spectra of the HPC (LC matrix), EFE, PEFE prepared in DMF containing no HPC, and

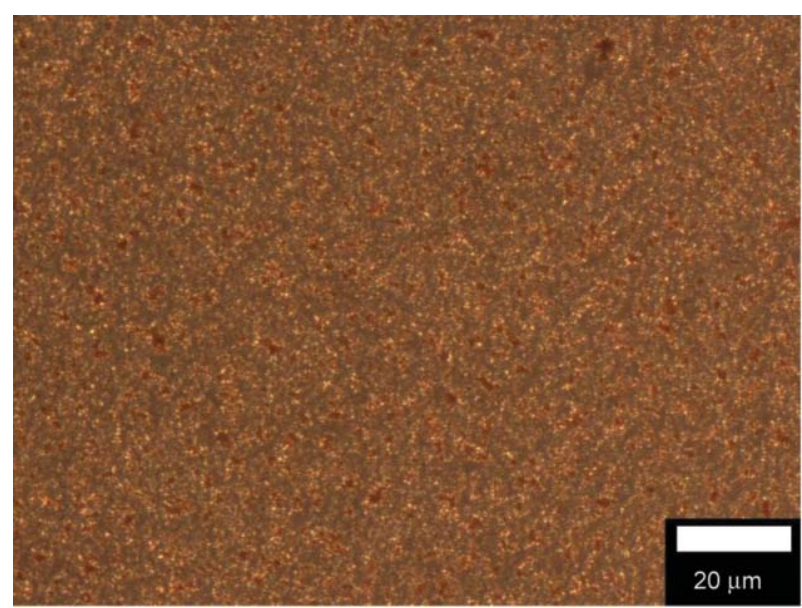

Figure 3. Polarizing optical microscopy (POM) image of PEFE film prepared in DMF with no HPC (hydroxypropyl cellulose) liquid crystal electrolyte solution $(200 \times)$

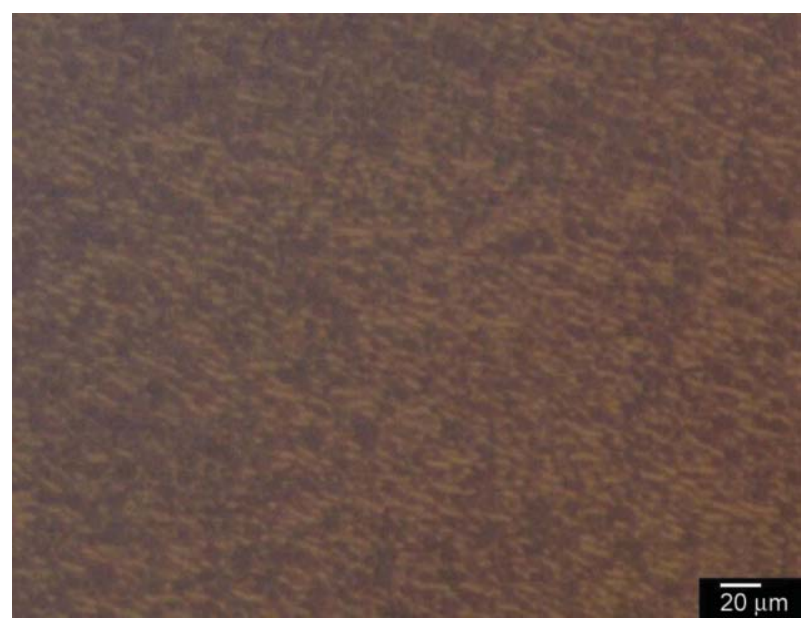

Figure 4. POM image of PEFE/HPC composite film $(500 \times)$

PEFE/HPC are shown in Figure 5. HPC shows O-H stretching vibration absorption at $3431 \mathrm{~cm}^{-1}$ due to hydroxyl group in the pyranose unit. Absorption band at around $3000 \mathrm{~cm}^{-1}$ is due to $\mathrm{CH}_{2}, \mathrm{CH}_{3}$ stretching vibration. The monomer (EFE) shows an absorption band at $1071 \mathrm{~cm}^{-1}$ due to $\mathrm{C}-\mathrm{O}-\mathrm{C}$ ring stretching of the EDOT unit. The corresponding polymer PEFE exhibits an absorption at $3422 \mathrm{~cm}^{-1}$ due to $\mathrm{O}-\mathrm{H}$ 


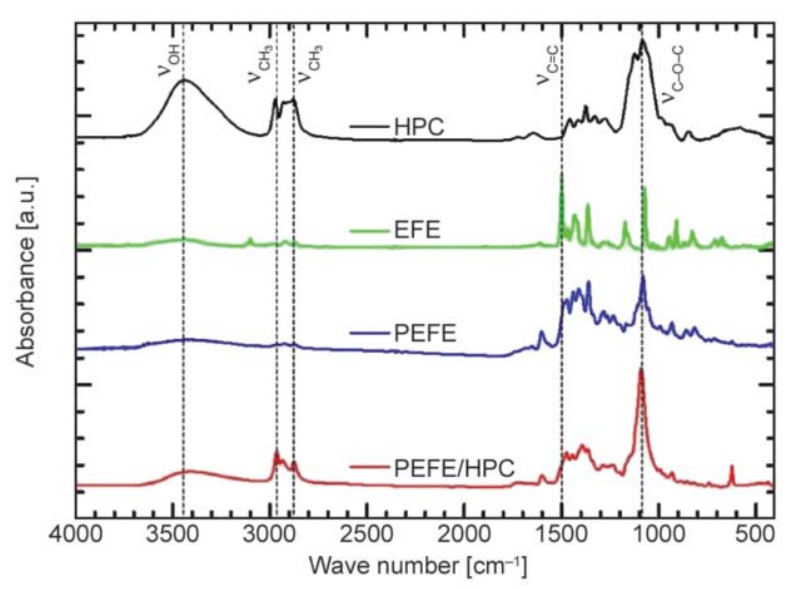

Figure 5. FT-IR spectra of hydroxypropyl cellulose (black line), monomer (green line), pure EFE film (blue line), and PEFE/HPC composite film (red line)

stretching of cellulose unit. This result indicates that PEFE/HPC is consisting of PEFE and HPC. PEFE entangles HPC, thus the HPC remained in the composite after washing. An absorption band at $1070 \mathrm{~cm}^{-1}$ is due to absorptions of $\mathrm{C}-\mathrm{O}-\mathrm{C}$ of cellulose and EDOT.

\subsection{UV-vis absorption}

In situ UV-vis absorption measurements were carried out by using cyclic voltammetry. UV-vis absorption spectra of PEFE/HPC at various applied potentials are shown in Figure 6. The polymer shows absorption band at $465 \mathrm{~nm}$ due to $\pi-\pi^{*}$ transition of the main chain. A new absorption band at $660 \mathrm{~nm}$ is due to polaron band (radial cations). Broad absorption at $>900 \mathrm{~nm}$ can be due to bipolarons (dications). The absorption intensity of the band increases with application voltages, indicating electrochemical oxidation (doping) is progressed with application of voltage. Contrary, reduction process (Figure 6b) displays decrease of absorption intensity due to polarons and

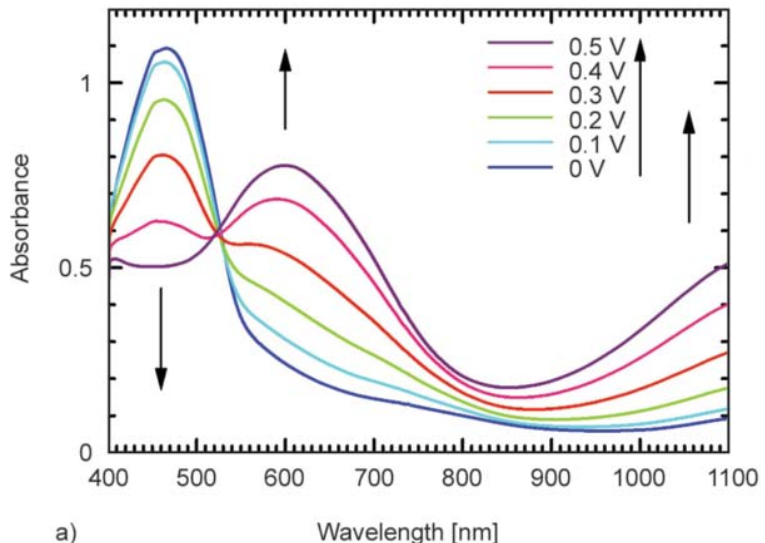

bipolarons. Absorption intensity at $465 \mathrm{~nm}$ is increased upon the electrochemical dedoping (reduction). Maximum absorption wavelength at the oxidized state and the reduced state are almost the same value in accordance with previously reported result [8].

UV-vis absorption spectra of PEFE film prepared in no HPC liquid crystal electrolyte solution (pure PEFE) and PEFE film prepared in HPC liquid crystal solution (PEFE/HPC) are shown in Figure 7. Pure PEFE and PEFE/HPC show absorption peaks at the same position (465 and $600 \mathrm{~nm}$ ).

This change in intensity is repeatable through electrochemical redox reaction. In other words, electrochemical doping-dedopoing is repeatedly achieved for the polymer composite. To confirm the repeatable electrochromic property, changes in absorption intensity at 465 and $660 \mathrm{~nm}$ upon repeating sequential application of voltage between 0 and $0.5 \mathrm{~V}$ (vs. $\mathrm{Ag} / \mathrm{Ag}^{+}$) at a scan rate of $30 \mathrm{mV} / \mathrm{s}$ for $650 \mathrm{~s}$ were examined (Figure 8). The intensity changes are complementary shape between $465 \mathrm{~nm}$ (absorption of main chain) and $600 \mathrm{~nm}$ (doping band).

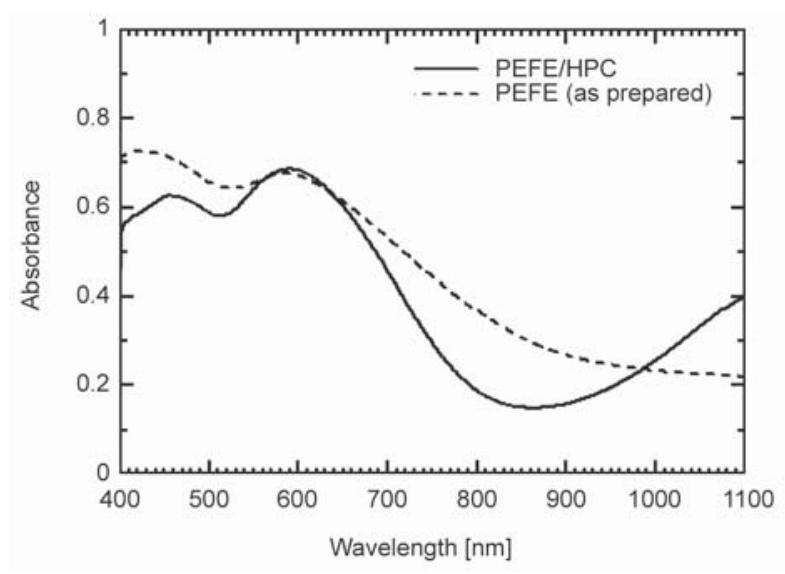

Figure 7. UV-vis absorption spectra of PEFE/HPC and PEFE

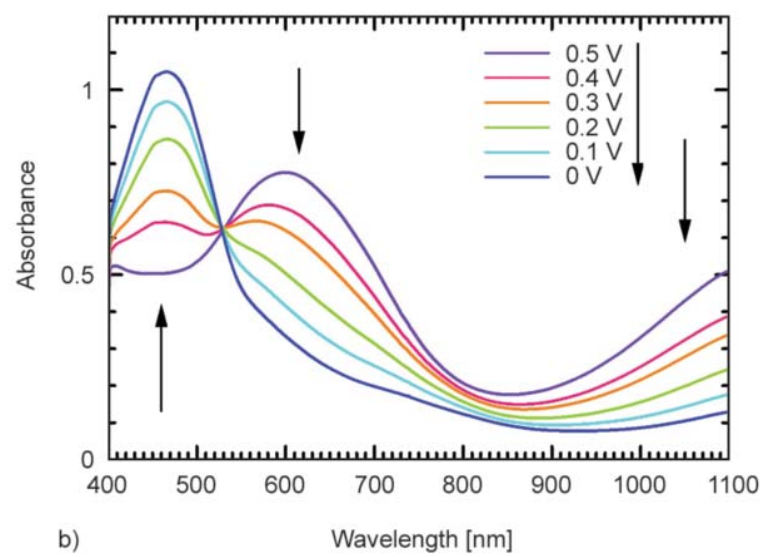

Figure 6. UV-vis absorption spectra of PEFE/HPC at various applied potentials (vs. $\mathrm{Ag} / \mathrm{Ag}^{+}$). Oxidation process (a) and reduction process (b) 


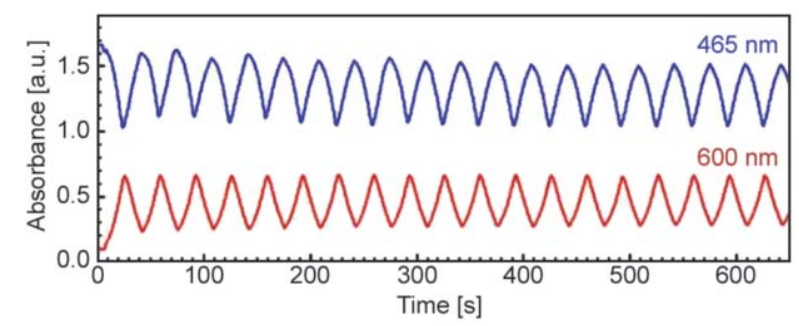

Figure 8. Time dependence of UV-vis absorption intensity at 465 and $660 \mathrm{~nm}$ of PEFE/HPC during repeated potential cycles. The potential was repeatedly applied between 0 and $0.5 \mathrm{~V}$ (vs. $\mathrm{Ag} / \mathrm{Ag}^{+}$) with a scan rate of $30 \mathrm{mV} / \mathrm{s}$ for $650 \mathrm{~s}$.

\subsection{Circular dichroism}

Circular dichroism (CD) absorption spectroscopy measurements were carried out for the PEFE film and the PEFE/HPC composite film (Figure 9). The PEFE film prepared in no HPC liquid crystal electrolyte solution shows no ellipticity. On the other hand, the PEFE film prepared in HPC liquid crystal electrolyte solution having chiral molecular order shows optical activity. The optical activity is derived from transcription of helical molecular order of HPC to the product.

Furthermore, the $\mathrm{CD}$ measurements for as-prepared sample (oxidized) and reduced sample were carried out. The CD spectra of the PEFE/HPC film are shown in Figure 10. The reduced sample was obtained with treatment of the polymer film with hydrazine vapor for $30 \mathrm{~min}$. Signal intensity of the CD band at $537 \mathrm{~nm}$ is increased with treatment of hydrazine, indicating reduction induced increase of ellipticity of the polymer composite.

\subsection{Possible structure}

A possible structure of PEFE/HPC is shown in Figure 11. HPC forms asymmetric $\beta$-pleated sheet

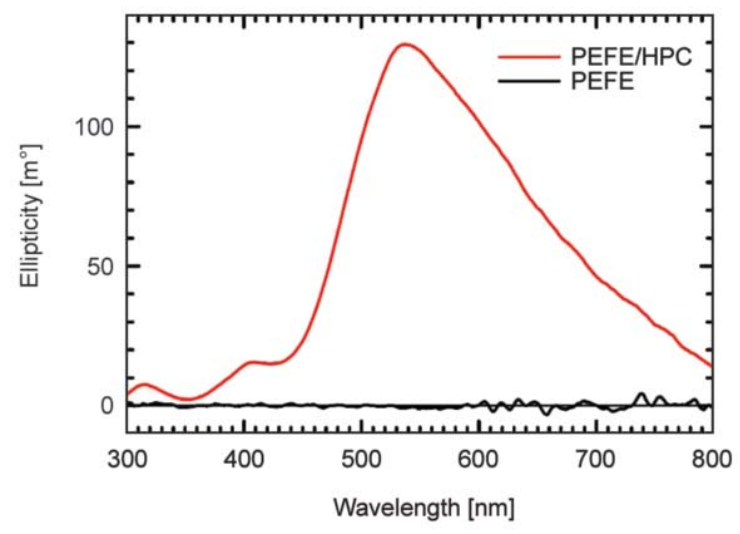

Figure 9. Circular dichroism (CD) spectra of PEFE/HPC and PEFE

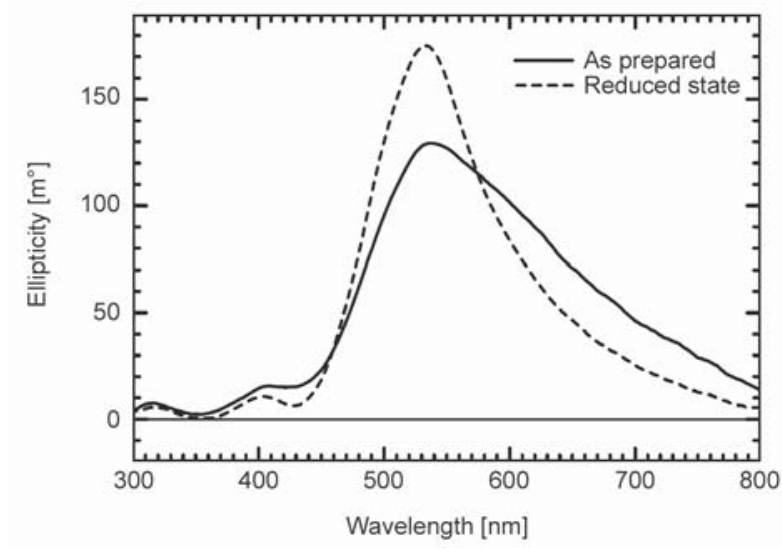

Figure 10. CD spectra of PEFE/HPC. Solid line: as prepared sample. Dashed line: reduced state.

structure in the molecular level, and the HPC aggregation forms helical structure in DMF at appropriate concentration to show cholesteric liquid crystal. The monomer is pre-oriented along the HPC prior to the polymerization reaction. Then, electrochemical polymerization is carried out and the polymer grows along the helical form of HPC to form 3-D helical structure. The product is polymer composite consisting of the $\pi$-conjugated polymer (PEFE) and the chiral non- $\pi$-conjugated polymer (HPC). The main chain of the PEFE enwinds HPC. Optical activity of the polymer film can be derived from formation of helical structure of the main chain or inter-chain helicity. In this case, mechanical asymmetric induction from HPC in molecular level and formation of helical aggregation with HPC occurred.

\section{Conclusions}

We achieved synthesis of electro-optically active polymer in the HPC liquid crystal. HPC/DMF system allows polymerization of hydrophobic monomer

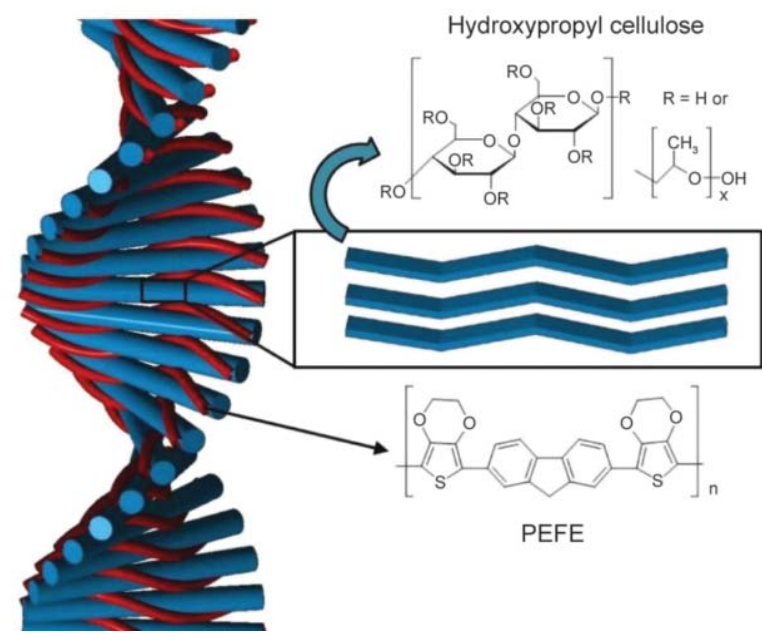

Figure 11. A possible structure of PEFE-HPC composite 
in lyotropic liquid crystal. The resultant composite film displays optical activity derived from cholesteric (chiral nematic) liquid crystal structure. PEFE prepared in a common organic solvent with no liquid crystalline order does not show such a liquid crystal like structure. This composite film shows repeatability in electrochromism.

\section{Acknowledgements}

We would like to thank Tsukuba Research Centre for Interdisciplinary Materials Science (TIMS). We would like to thank Chemical Analysis Center of University and Glass Work Shop of University of Tsukuba.

\section{References}

[1] Krishnamoorthy K., Ambade A. V., Kanungo M., Contractor A. Q., Kumar A.: Rational design of an electrochromic polymer with high contrast in the visible region: Dibenzyl substituted poly(3,4-propylenedioxythiophene). Journal of Materials Chemistry, 11, 2909-2911 (2001).

https://doi.org/10.1039/b108654e

[2] Gaupp C. L., Welsh D. M., Reynolds J. R.: Poly(ProDOT$\mathrm{Et}_{2}$ ): A high-contrast, high-coloration efficiency electrochromic polymer. Macromolecular Rapid Communications, 23, 885-889 (2002).

https://doi.org/10.1002/15213927(20021001)23:15<885::AID-MARC885>3.0.CO;2-X

[3] Otero L., Sereno L., Fungo F., Liao Y-L., Lin C-Y., Wong K-T.: Synthesis and properties of a novel electrochromic polymer obtained from the electropolymerization of a 9,9'-spirobifluorene-bridged donor-acceptor (D-A) bichromophore system. Chemistry of Materials, 18, 3495-3502 (2006).

https://doi.org/10.1021/cm0605264

[4] Gaupp C. L., Zong K., Schottland P., Thompson B. C., Thomas C. A., Reynolds J. R.: Poly(3,4-ethylenedioxypyrrole): Organic electrochemistry of a highly stable electrochromic polymer. Macromolecules, 33, 1132 1133 (2000)

https://doi.org/10.1021/ma9916180
[5] Matsumura A., Fan Y., Goto H.: Asymmetric electrochemical polymerization in cholesteric liquid crystalline media: Effect of isomeric structures of chiral inducers containing bornyl group. Synthetic Metals, 202, 157-164 (2015).

https://doi.org/10.1016/j.synthmet.2015.01.019

[6] Kawabata K., Takeguchi M., Goto H.: Optical activity of heteroaromatic conjugated polymer films prepared by asymmetric electrochemical polymerization in cholesteric liquid crystals: Structural function for chiral induction. Macromolecules, 46, 2078-2091 (2013).

https://doi.org/10.1021/ma400302j

[7] Nie G., Yang H., Chen J., Bai Z.: A novel high-quality electrochromic material from 3,4-ethylenedioxythiophene bis-substituted fluorene. Organic Electronics, 13, 2167-2176 (2012). https://doi.org/10.1016/j.orgel.2012.05.055

[8] Wang A., Kawabata K., Kawashima H., Goto H.: Synthesis of a pyrimidine-based new chiral inducer for construction of cholesteric liquid crystal electrolyte solution and its electrochemical polymerization, and stimulated emission like interference. Polymer, 54, 38213827 (2013).

https://doi.org/10.1016/j.polymer.2013.04.053

[9] Eguchi N., Kawabata K., Goto H.: Chiral-electroactive low-bandgap polymer composite. Journal of Materials Science and Chemical Engineering, 5, 1-10 (2016). https://doi.org/10.4236/msce.2017.52001

[10] Eguchi N., Kawabata K., Goto H.: Electrochemical polymerization of 4,4-dimethyl-2,2'-bithiophene in concentrated polymer liquid crystal solution. Journal of Materials Science and Chemical Engineering, 5, 64-70 (2017). https://doi.org/10.4236/msce.2017.52007

[11] Kawabata K., Goto H.: Periodic structure in a fluorenebased polymer prepared by electrochemical polymerization. Chemistry Letters, 38, 706-707 (2009). https://doi.org/10.1246/cl.2009.706

[12] Vshivkov S. A., Rusinova E. V., Galyas A. G.: Phase diagrams and rheological properties of cellulose ether solutions in magnetic field. European Polymer Journal, 59, 326-332 (2014). https://doi.org/10.1016/j.eurpolymj.2014.07.042 